\title{
Influences of Groove Depth on the Coupling of Small Tip Clearance and Circumferential Grooves
}

\author{
Tianye $\mathrm{Ji}^{\text {a }}$, Xie Fang, Youjun Wang and Tao Yi \\ Xi'an Research Institute of Hi-tech, Xi'an 710025, Shaanxi, People's Republic of China \\ a15594809968@163.com
}

\begin{abstract}
Coupling of small tip clearance and circumferential grooves of axial-flow compressors significantly improves the stability margin, as well as pressure ratio and efficiency. Different groove depth under coupling of small tip clearance and circumferential grooves has been designed for the isolate subsonic NPU-rotor based. Moreover, influences and mechanism of groove depth on the coupling of small tip clearance and circumferential grooves have been further studied by utilizing a numerical simulation calculation method. The results showed that the role of casing treatment reduced driving force of generating leakage action, postponed negative effects intersecting incoming flow with tip clearance flow and improved flow field of tip passages by sucking leakage flow into the groove.
\end{abstract}

Keywords: Transonic compressor; Numerical simulations; Circumferential groove casing treatment

\section{Introduction}

A The reduction of small tip clearance in axial-flow compressors can improve stable operating range of compressors, but seldom improve pressure ratio, or even reduce pressure ratio [1-3]. Some domestic scholars have conducted deep study on the casing treatment. It can be found that reasonable circumferential groove design can dramatically enhance stability of compressors, but efficiency will be reduced in varying degrees [4-6]. In addition, some overseas scholars also have proposed similar problems and opinions [7]. Liu Le [8] carried out the experimental study on single circumferential grooves and thought that single front-end grooves can restrain unstable fluctuation of leakage flow, but influences of rear-end single grooves are not obvious. Based on the above-mentioned studies, the author thinks that single circumferential groove structure has a higher requirement for its position and groove depth, so it is necessary to carry out an in-depth study on stall margin and stability enhancement of multi-groove structure in compressors. Single circumferential grooves with different groove depth under small clearance are designed for the isolate subsonic NPU-rotor based, showing main design parameters in Table 1.

Table 1 Main design parameters of rotor

\begin{tabular}{c|c}
\hline Design parameters & Parameter Values \\
\hline Number of blades & 30 \\
Design isentropic adiabatic efficiency & 0.905 \\
Design rotate speed(rpm) & 15200 \\
Design total pressure ratio & 1.249 \\
Tip relative Mach number & 0.78 \\
Aspect ratio of blades & 1.94 \\
Inlet hub-to-tip ratio & 0.61 \\
Tip radial clearance $(\mathrm{mm})$ & 0.4 \\
Tip tangential velocity $(\mathrm{m} / \mathrm{s})$ & 237 \\
Solidity & 0.96 \\
\hline
\end{tabular}

\section{Different Groove Depth Design of Subsonic Compressors}

Under a half of design clearance conditions, two circumferential grooves with different groove depth are designed in Figure 1, and main geometrical parameters are shown in Table 2. Tip clearance is a half of design clearance. Circumferential grooves set up H-type gridding. The groove gridding is distributed into $109 \times 17 \times 9$ (circumference $\times$ radial direction $\times$ axial direction). Groove bottom and 
groove side have thermal insulated surface and non-slipping conditions, as well as apply non-matching gridding boundary conditions with mainstream interface. Considering fluid viscidity, boundary condition is the solid wall boundary. The grid point of the first layer has the distance of $2 \times 10^{-6} \mathrm{~m}$ from the solid wall, so as to ensure $\mathrm{y}^{+}<2$.

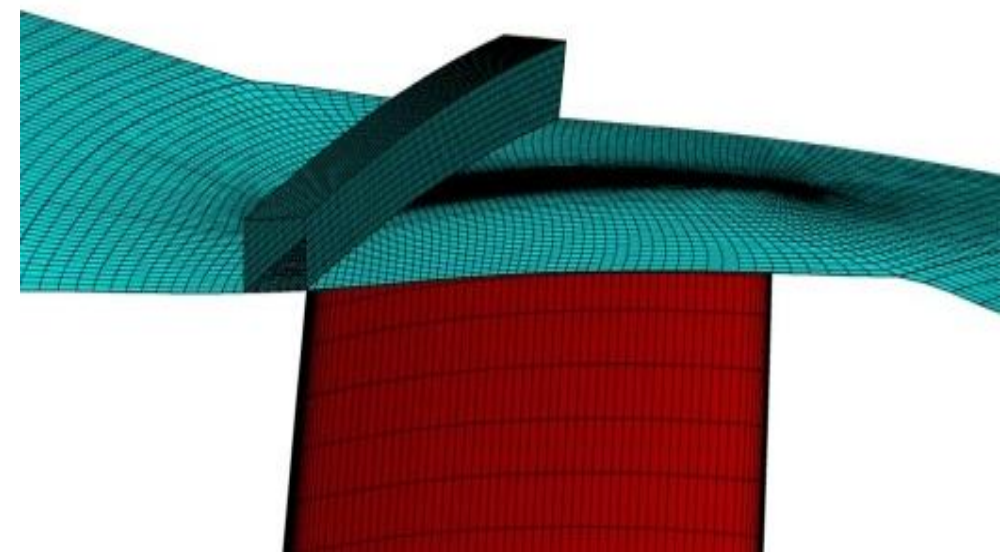

Fig. 1 Structural design diagram of single circumferential grooves

Table 2 Main geometrical parameters of single circumferential grooves

\begin{tabular}{c|c|c|c|c}
\hline Process form & $\begin{array}{c}\text { Solid-Experimen } \\
\mathrm{t}\end{array}$ & Solid-Experiment & Treament3 & Treament6 \\
\hline Depth & $/$ & $/$ & 3 & 6 \\
\hline Width & $/$ & $/$ & 3 & 3 \\
\hline $\begin{array}{c}\text { The distance from } \\
\text { leading edge to } \\
\text { treatment center }(\mathrm{mm})\end{array}$ & $/$ & $/$ & 1.5 & 1.5 \\
\hline Tip clearance & $\begin{array}{c}\text { design-clearance } \\
(0.4 \mathrm{~mm})\end{array}$ & $\begin{array}{c}\text { half design-clearance } \\
(0.2 \mathrm{~mm})\end{array}$ & $\begin{array}{c}\text { half } \\
\text { design-clearance }\end{array}$ & $\begin{array}{c}\text { half design-clearance } \\
(0.2 \mathrm{~mm})\end{array}$ \\
\hline
\end{tabular}

\section{Overall Performance}

Figure 1 is the compressor performance curve of coupling tip clearance and casing treatment and displays numerical calculation characteristic curves, including experimental characteristics of clearance solid wall, characteristics of a half of clearance solid wall, single-groove casing treatment of a half of clearance ( $3 \mathrm{~mm}$, it is named as treatment-3mm), and single-groove casing treatment of a half of clearance $(6 \mathrm{~mm}$, it is named as treatment $-6 \mathrm{~mm})$.
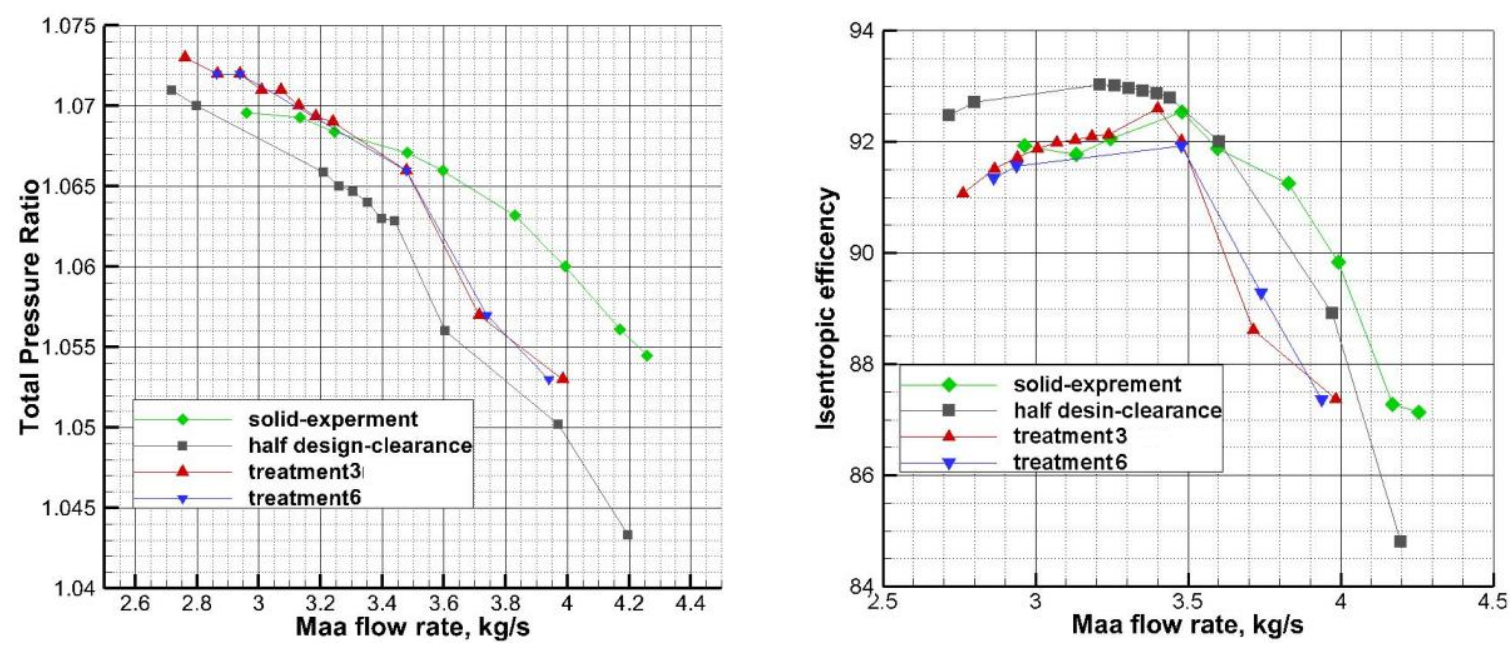

Fig. 2 The compressor performance curve between coupling of tip clearance and casing treatment of subsonic compressors 
By analyzing Figure 2, the following conclusions can be drawn: (1) stability margin of a half of design clearance is improved by making a comparison with single-groove coupling and untreated smooth wall. Particularly, improved quantity of stability margin for shallow grooves reaches $7.5966 \%$, and improved quantity of peak efficiency is $0.09 \%$; (2) Peak efficiency of compressor efficiency is slightly higher than near stall point of untreated smooth wall, and the entire efficiency is greater than untreated smooth wall's entire efficiency; (3) Under a half of design clearance, pressure ratio of casing treatment is improved by comparing with untreated smooth wall. The above-mentioned conclusions indicate that using single-groove casing treatment and tip clearance coupling can improve stall margin dramatically without reducing efficiency and pressure ratio. Meanwhile, the above-mentioned experiment and numerical simulation show that the conclusions and analyses of stall margin are correct.

\section{Analysis of Stability Enhancement}

Figure 3, 4 and 5 are relative Mach number cloud of 99\% blade height, entropy distribution cloud and tip clearance leakage streamline chart for solid-wall casing treatment, treatment-6mm and treatment-3mm(two kinds of groove depth), respectively. Mass flow rate of compressors under three states is approximately equal. Untreated smooth wall is kept in the near stall condition. It can be observed from Figure 3 that incoming flow of untreated smooth wall and tip leakage flow intersecting plane are parallel to the leading edge, and the entire passage is almost blocked by low-energy clusters. Figure 4 and 5 display intersecting plane of incoming flow and tip clearance leakage flow is backward obviously, because there is casing treatment, corresponding to Figure 4(Surface Static Pressure Distribute Diagram of 99\% Blade Height). The intersecting position exactly is the position increasing static pressure difference. Thus, even if their groove depth is different, but entropy production displays the same position of entropy production, indicating that the position of circumferential grooves has an important influence on incoming flow and intersection position of tip leakage flow, but groove depth has no big influences on the intersecting position. However, the casing treatment in the same position brings about obviously different losses for different groove depth, resulting in diverse degree of low-energy clusters' blocking passages. In the Figure 5, areas of low-energy clusters are smaller, and the casing treatment can operate under the higher outlet backpressure. Thus, it can acquire higher improved quantity of stall margin. Too large groove depth reduces driving force of flowing leakage, resulting in reducing secondary clearance leakage.

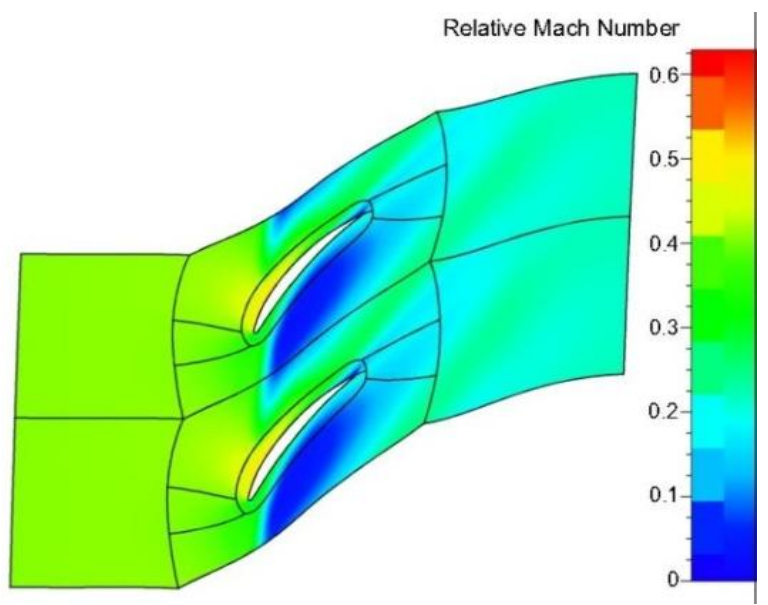

(a)The relative Mach number contour 


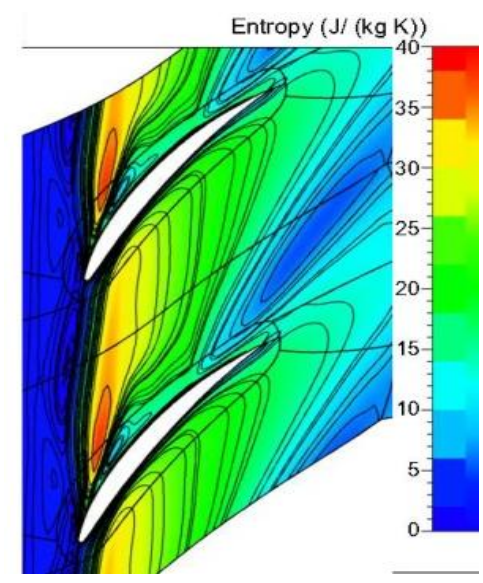

(b)The entropy contour

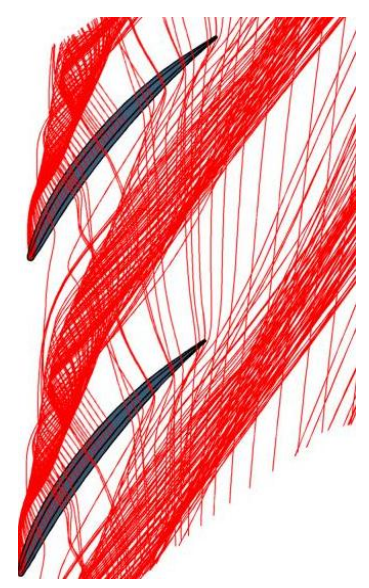

(c)The streamlines released in tip region

Fig.3 Relevant contours of smooth wall with half design-clearance at $99 \%$ span

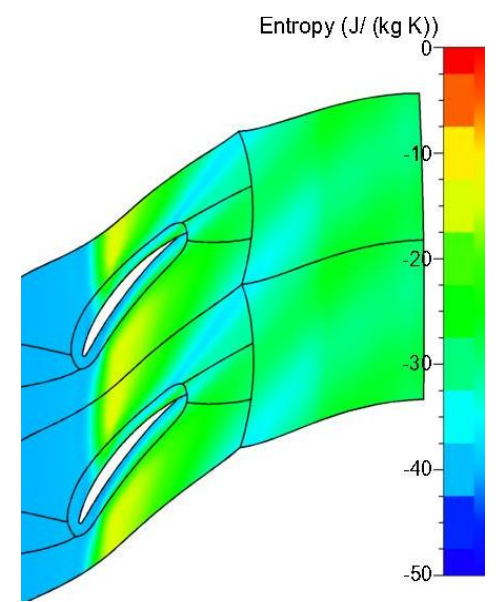

(a)The relative Mach number contour

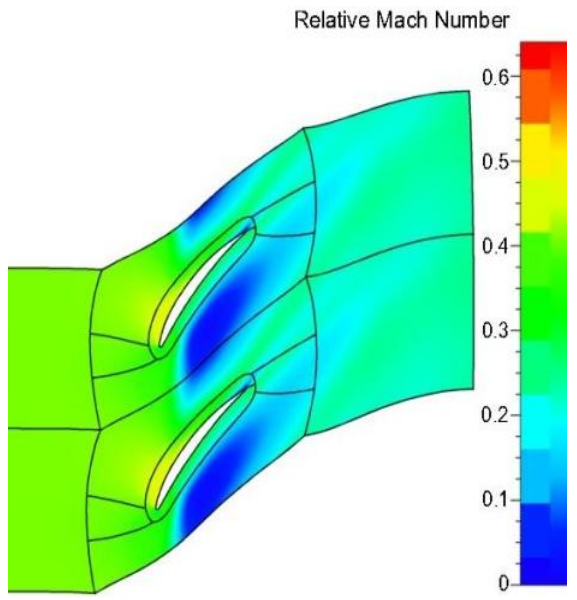

(b)The entropy contour

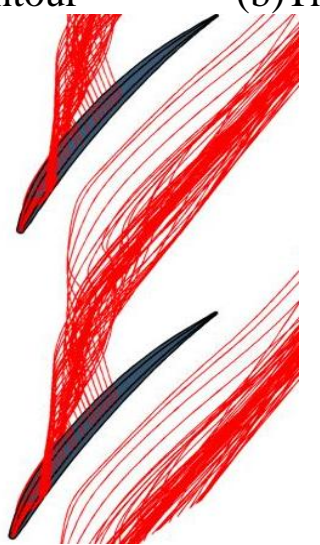

(c)The streamlines released in tip region

Fig.4 Relevant contours of treatment-6mm with half design-clearance at $99 \%$ span

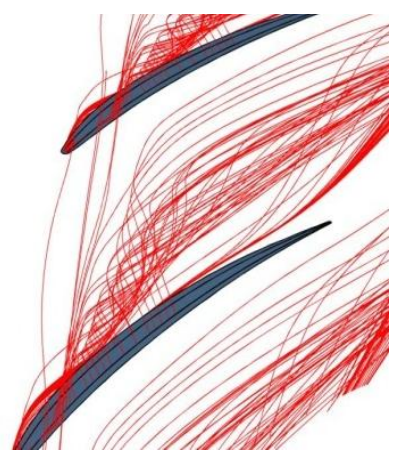

(a)The relative Mach number contour 


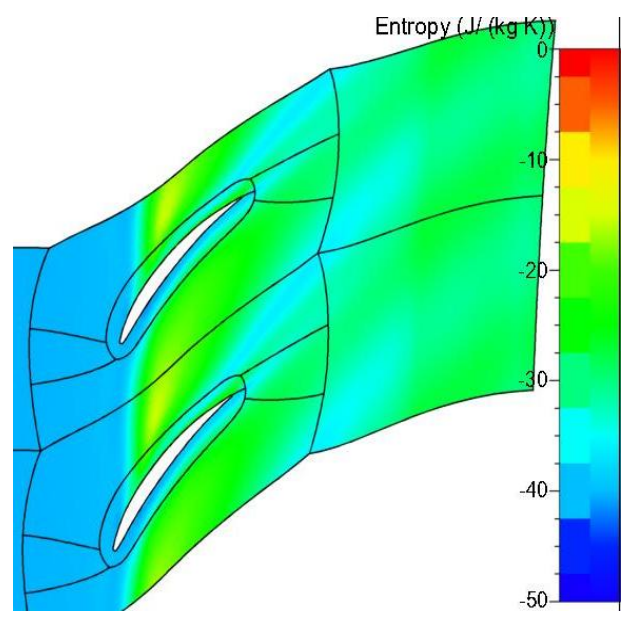

(b) The entropy contour

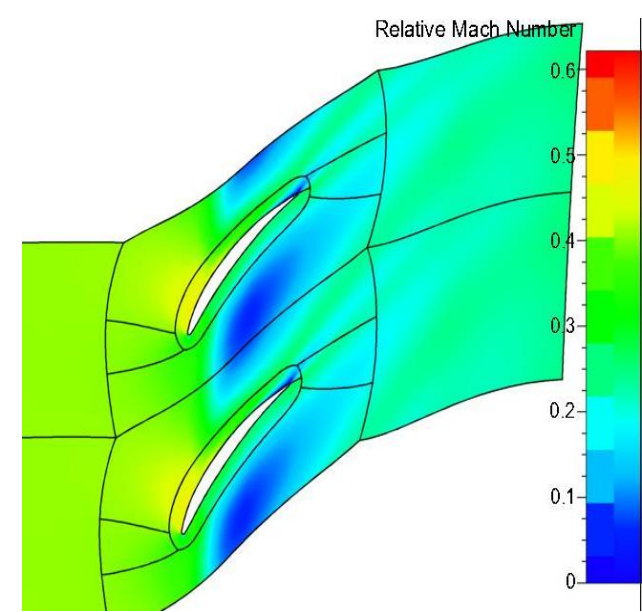

(c)The streamlines released in tip region

Fig. 5 Relevant contours of treatment-3mm with half design-clearance at $99 \%$ span

Figure 6 and 7 provides relative speed of meridian plane and distribution diagram of relative total pressure of untreated smooth wall and two casing treatments (3-m groove depth and 6-m groove depth). Mass flow rate of compressors under three states is approximately equal. Untreated smooth wall is kept in the near stall condition. The curve $L$ in the figure stands for the intersecting plane curve of relative high and low-pressure total pressure (averaged by circumference). The figure shows that axial position of curve $\mathrm{L}$ has no big difference, but untreated smooth wall locates in lower blade position, indicating that downwash tendency of clearance leakage flow is more obvious, and backflow phenomenon in clearance is serious. The downwash tendency of deep grooves in two casing treatments is greater than corresponding shallow grooves. There is obvious vortex in single grooves.

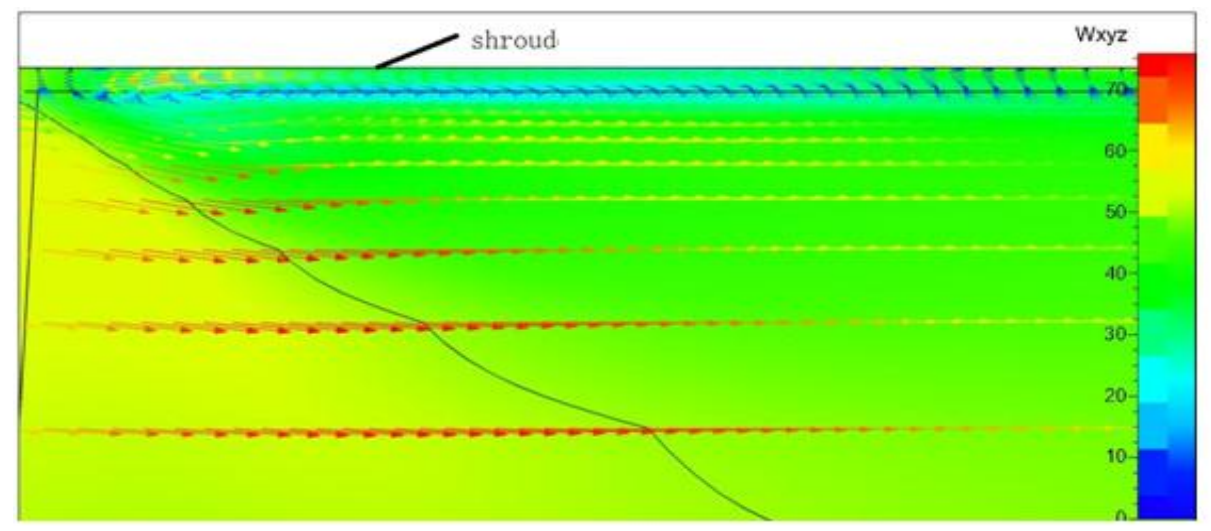

Fig.6 Meridional velocity and total pressure contours of smooth wall

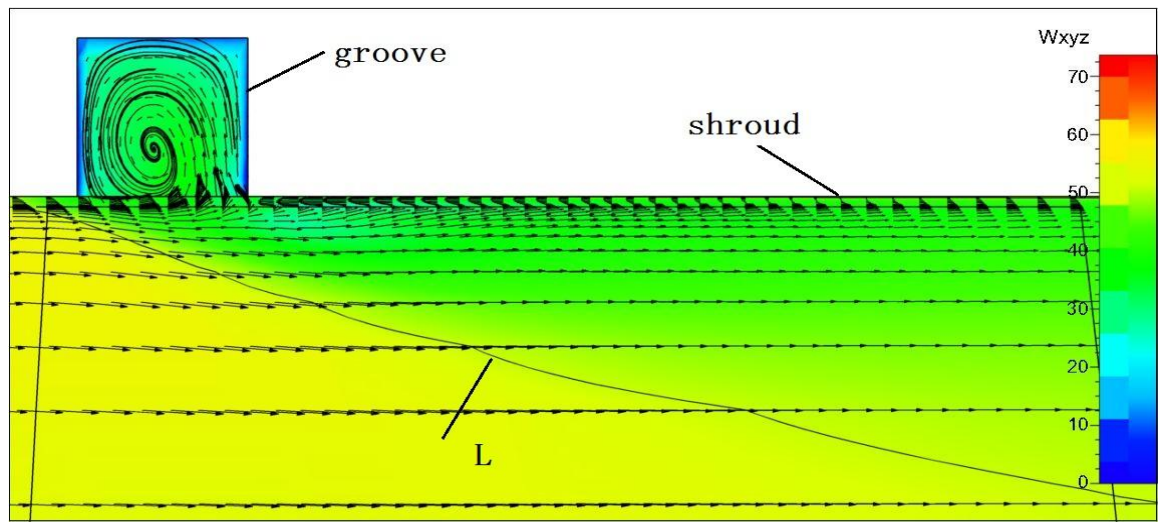

Fig.7 Meridional velocity and total pressure contours of treatment-3mm 


\section{Conclusion}

The paper mainly studied changes and related mechanism of compressor characteristics in coupling of small clearance and circumferential grooves. Nowadays, seldom scholars study the above-mentioned topic. Based on the isolate subsonic NPU-rotor, the paper discussed stability enhancement mechanism of circumferential grooves and discussed influences of groove depth on compressor performance by conducting flow field analysis through numerical simulation. The follow conclusions can be drawn from the above-mentioned research:

(1) Downwash phenomenon of airflow in passages was restrained, tip accumulations of low-energy clusters were reduced, flow capacity was enlarged effectively, and stable operating ranges of compressors was enlarged under the function of circumferential grooves.

(2) Grooves with too large depth also induced strong internal swirling flow and thus large loss generation. Effective control of groove depth not only played the function of radial suction, but also avoided from redundant energy losses as many as possible. The important approach to enhance effectiveness of casing treatment is reasonable design of groove depth.

\section{References}

[1]. Denton, J. D., 1993, "Loss Mechanisms in Turbomachines," ASME J. Turbomach, 115, pp. 621-656. [2] Adamczyk, J. J., Celestina, M. L., and Greitzer, E. M., 1993, "The Role of Tip Clearance in High-Speed Fan Stall," ASME J. Turbomach., 115, pp. 28-39.

[2]. Bergner, J., and Hennecke, D. K., 2003, "Experimental Study of Stall Inception of a Single-Stage Transonic Compressor," Proceedings of the 16th Symposium Air Breathing Engines, Cleveland, OH, August 31-September 5, Paper No.ISABE-2003-1081.

[3]. Biela, C., Muller, M. W., Schiffer, H., and Zscherp, C., 2008, "Unsteady Pressure Measurement in a Single Stage Axial Transonic Compressor Near the Stability Limit," ASME Paper No. GT2008-50245.

[4]. Reid, L.and Moore, R. D., 1978. Design and overall performance of four highly-loaded, high-speed inlet stages for an advanced, high-pressure-ratio core compressor[R]. NASA.TP-1337.

[5]. XIE Fang, CHU Wu-li, ZHANG Hao-guan, Influence shock waves leakage vortex boundary layer separation interaction in a single- stage transonic axial compressor [J], 2012. 27(2), 425-430.

[6]. Suder, K. L., and Celestina, M. L., 1996, "Experimental and Computational Investigation of the Tip Clearance Flow in a Transonic Axial Compressor Rotor,” ASME J. Turbomach., 118(2), pp. $218-229$.

[7]. Vo, H. D., Tan, C. S., and Greitzer, E. M., 2008, “Criteria for Spike Initiated Stall,” ASME J. Turbomach., 130(1), p. 011023. 